ISSN electrónico: 2445-1355

DOI: https://doi.org/10.14201/fj2021623948

\title{
ESTUDIO DE EVOLUCIÓN DE CONSUMO \\ DE ANTIBIÓTICOS Y PATRÓN DE RESISTENCIAS \\ EN UNA UNIDAD DE CUIDADOS INTENSIVOS DESDE EL AÑO 2013-2018
}

\section{Study on the Evolution of Antibiotic Consumption and Resistance Pattern in an Intensive Care Unit from 2013-2018}

Lara SANCHO ROMO*; Juan José ORTIZ DE URBINA GONZÁLEZ**; Julio Antonio VALDUEZA BENEÍTEZ***

* Facultad de Farmacia. Universidad de Salamanca.

Correo-e: larasanchoromo@gmail.com

* Jefe de Servicio de Farmacia. Complejo Asistencial Universitario de León. Correo-e: jortiz@saludcastillayleon.es

*** Servicio de Farmacia. Hospital Comarcal de El Bierzo (C/ Médicos sin Fronteras, 7. 24404 Ponferrada, León).

Correo-e: jvaldueza@saludcastillayleon.es

RESUMEN: La aparición y la diseminación de las resistencias a antibióticos son el resultado de un proceso de selección adaptativa en respuesta al uso de antibióticos denominada presión antibiótica. La presión antibiótica no solo facilita la expansión de los mutantes resistentes, sino que la exposición a dosis subletales de antibiótico puede favorecer la mutación de especies resistentes a distintas familias de antibióticos. Esto adquiere una gran importancia en el medio hospitalario y especialmente en las unidades de cuidados intensivos (UCI), por lo que es crucial controlar las distintas cepas endémicas del 
hospital y ajustar la dosis de antibioticoterapia empleada en cada paciente. Por ello se iniciaron a nivel nacional los Programas de Optimización de uso de Antimicrobianos (PROA). En este trabajo se realiza un análisis de la situación de partida de la UCI del Hospital de El Bierzo, evaluando consumo de antibióticos por familias y principios activos, cepas aisladas y resistencias de las cepas aisladas, para evaluar la necesidad de implantación de un equipo PROA.

Palabras clave: resistencias; antibióticos; UCI; PROA.

ABSTRACT: The appearance and spread of antibiotic resistance is the result of an adaptive selection process in response to the use of antibiotics, called antibiotic pressure. Antibiotic pressure not only facilitates the expansion of resistant mutants, but exposure to sublethal doses of antibiotics can favor the mutation of species resistant to different families of antibiotics. This acquires great importance in the hospital environment and especially in intensive care units (ICU), so it is crucial to control the different endemic strains of the hospital, and to adjust the dose of antibiotic therapy used in each patient. For this reason, the Antimicrobial Use Optimization Programs (PROA) were initiated at the national level. In this work, an analysis of the initial situation of the ICU of the Hospital de El Bierzo is carried out, evaluating the consumption of antibiotics, by families and active principles, isolated strains, and resistance of the isolated strains, to evaluate the need for implantation of a PROA team.

Keywords: resistance; antibiotics; ICU; PROA.

\section{Introducción}

Nuestra microbiota es fundamental para numerosas funciones fisiológicas e inmunológicas, y las bacterias ambientales son los cimientos sobre los que se sustenta la vida. En condiciones normales las bacterias resistentes tienen menos capacidad de propagación que las no resistentes, pero en situaciones de exposición a antibiótico (ATB) sucede lo contrario. La aparición y la diseminación de las resistencias adquiridas a ATB son el resultado de un proceso de selección adaptativa en respuesta al uso de ATB denominada presión antibiótica.

Se ha demostrado que la presión antibiótica sobre una población bacteriana no solo conduce a una expansión de los mutantes resistentes a aquel, sino que la exposición de las bacterias a dosis subletales puede dar lugar a mutantes resistentes 
a ATB de familias diferentes, debido a la inducción de mutagénesis mediada por radicales libres, generada por el estrés frente al ATB sin que este llegue a ser letal. Sin embargo, esta relación es compleja, no siempre bien conocida, y con frecuencia condicionada por otros factores no bien establecidos (Contreras, 2018; Lázaro, Oteo, 2006).

En el medio hospitalario, las unidades de cuidados intensivos (UCI) son los escenarios más propicios para el desarrollo de infecciones intrahospitalarias por bacterias resistentes a antibióticos, debido a la alta prevalencia de la infección nosocomial. Por ello es necesario garantizar un tratamiento antibiótico adecuado, conociendo el microorganismo y su perfil de resistencia, ya que no tratar eficazmente la infección se verá reflejado en un incremento de la estancia y la mortalidad del paciente (Pallares, Martínez, 2012). Para ello, a nivel nacional e internacional, se están promoviendo medidas de control de uso de antibióticos, como son la implantación de Programas de Optimización de uso de Antimicrobianos (PROA) tanto en hospitales como equipos específicos de UCI, que es uno de los objetivos del Plan Nacional frente a Resistencias a Antibióticos.

El PROA es un equipo multidisciplinar encaminado a evitar el uso innecesario o inapropiado de antibióticos y realizar vigilancia epidemiológica de la flora del servicio u hospital objeto de estudio (Generalitat de Cataluya, 2018).

La prescripción de antimicrobianos en los pacientes de la UCI es una de las estrategias más frecuentes debido a las patologías propias del paciente y al riesgo de infección intrahospitalaria (Vega et al., 2015), esto, sumado a la dificultad a la hora de diferenciar entre el Síndrome de Respuesta inflamatoria Sistémica (SRIS), de origen infeccioso y no infeccioso, hace que la prescripción empírica de un tratamiento sea un factor crítico en la evolución de dicho paciente, para lo cual es imprescindible conocer la flora endógena (Rodríguez-Baño et al., 2012).

El objetivo de este estudio será la compilación de datos sobre consumo de antibióticos y flora bacteriana en la UCI del Hospital Universitario de El Bierzo, que sirva como base para el desarrollo de un equipo PROA-UCI.

\section{MATERIALES y MÉTODOS}

- Tipo de estudio: observacional, descriptivo, longitudinal y retrospectivo.

- Periodo: enero de 2013 a diciembre de 2018.

- Lugar: UCI del Hospital de El Bierzo, hospital de segundo nivel, con 420 camas en total, 13 en la UCI.

- Fuente de datos: los registros informáticos del módulo de gestión de Farmatools ${ }^{\circledR}$ del Servicio de Farmacia a la UCI; los datos microbiológicos, reportados por el Servicio de Microbiología; los datos de las estancias, por la Gerencia del Hospital, y los datos de 2013-2018 del ENVIN-UCI. 
- Registro y procesamiento: se realizó una clasificación de los fármacos según el código ATC propuesto por la OMS (WHOCC, 2019), empleando para ello una hoja de cálculo de OpenOffice Calc, donde se reflejaron los consumos anuales por unidades dispensadas, a partir de las cuales se calcularon los gramos consumidos para obtener las DDD por 100 estancias de cada principio activo según la siguiente fórmula:

$\mathrm{DDD} / 100$ ESTANCIAS $=($ CONSUMO $(\mathrm{g}) / \mathrm{DDD}(\mathrm{g})) *(100 /$ ESTANCIAS)

A partir de los datos obtenidos se obtuvieron porcentajes anuales de uso, variación porcentual y otros datos reflejados en el apartado de resultados.

\section{Resultados}

Se han recogido los datos de consumo de una media de 38 principios activos, dependiendo del año, con distintas especialidades farmacéuticas de 20 grupos terapéuticos. El consumo global de cada año se incluye en la Figura 1, reflejando la tendencia.

\section{DDD TOTALES POR AÑO}

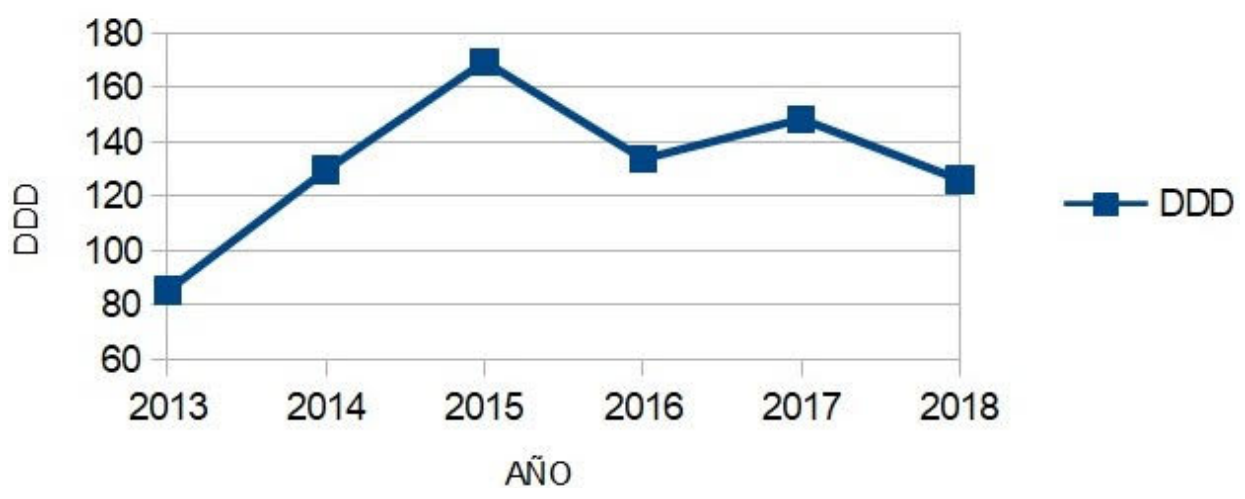

Figura 1: Evolución de las DDD/100 estancias totales consumidas entre 2013-2018.

El consumo medio de antimicrobianos entre los seis años fue de 131,96 DDD/100 estancias, con un incremento porcentual del 11,51 \% desde 2013 a 2018.

Por cada año se analizó el consumo porcentual de DDD según el grupo terapéutico, quedando patente que todos los años los antibióticos más consumidos 
fueron betalactamas (J01AC), betalactamas con inhibidores de betalactamasas (J01AR), carbapenems (J01DH), aminoglucósidos (J01GA), quinolonas (J01MA) y otros antibióticos (J01XX).

También se agruparon los consumos según se empleen para el tratamiento de infecciones por GRAM(+) o GRAM(-), así como los principales antiinfecciosos de amplio espectro y activos frente a anaerobios y antimicóticos.

Se estudiaron las líneas de tendencia, que reflejan aumento de consumo en antimicrobianos para GRAM(+), de cloxaciclina (J01CF02) y linezolid (J01XX08), el cual es un antibiótico de uso restringido. Respecto a los glucopéptidos destacar que casi la totalidad de las DDD consumidas son de vancomicina (J01XA01).

Para los antimicrobianos que se emplean frente a GRAM(-), se siguió la misma metodología, apreciando tendencia de aumento de consumo en carbapenems (J01DH), amoxicilina/clavulánico (J01CR02), piperacilina/tazobactam (J01CR05) y cefalosporinas de 3..$^{a}$ y.$^{a}$ generación (J01DD y J01DE).

La valoración por principio activo indicó que, de los aminoglucósidos, la amikacina era la más consumida; el meropenem y el imipenem en los carbapenemes, y la cefotaxima dentro de las cefalosporinas.

Respecto a otros antimicrobianos y antimicóticos, se apreciaron en general tendencias constantes o mínimamente ascendentes para todos excepto para las fluoroquinolonas, que descienden mínimamente, siendo levofloxacino el más consumido, y antimicóticos, que tienen un valor muy alto en 2015 para anfotericina, alterando la tendencia general.

También se debe comentar la tendencia ascendente del cotrimazol, hasta 2018 que su uso se redujo notablemente.

Los microorganismos fueron aislados de muestras de sangre, orina, esputo, aspirado bronquial, úlcera cutánea, catéter intravascular, absceso, aspirado traqueal, bilis, drenaje, exudado de herida, piel y rectal, frotis nasal y rectal y líquido ascítico, cefalorraquídeo y peritoneal.

Las proporciones de la flora aislada se refleja en la Figura 2 y en la Figura 3 se puede apreciar la evolución de las cepas resistentes más significativas de estos microorganismos. 


\section{PROPORCIÓN DE CEPAS AISLADAS 2013-2018}

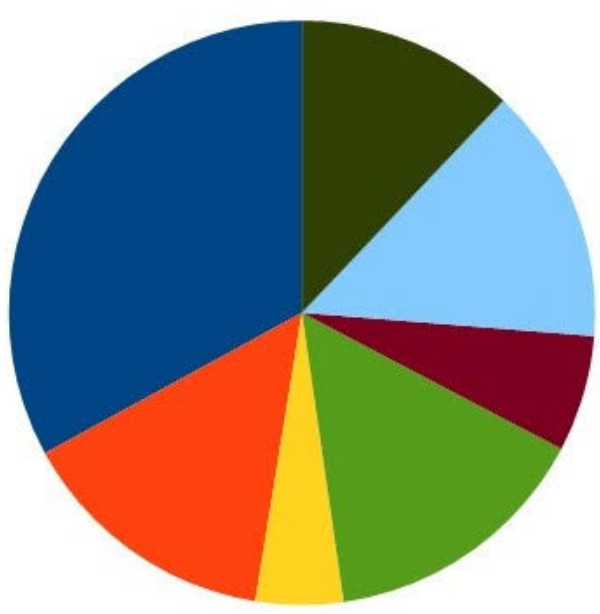

- Escherichia coli (33\%)

Klebsiella pneumiae (14\%)

Enterobacterias (5\%)

- Pseudomonas aeruginosa (15\%)

- Enterococo faecalis $(6 \%$

Enterococo faecium (14\%)

- Staphylococcus aureus

(12\%)

Figura 2: Proporción de cepas aisladas.

\section{PROGRESIÓN DE BACTERIAS RESISTENTES POR AÑO}

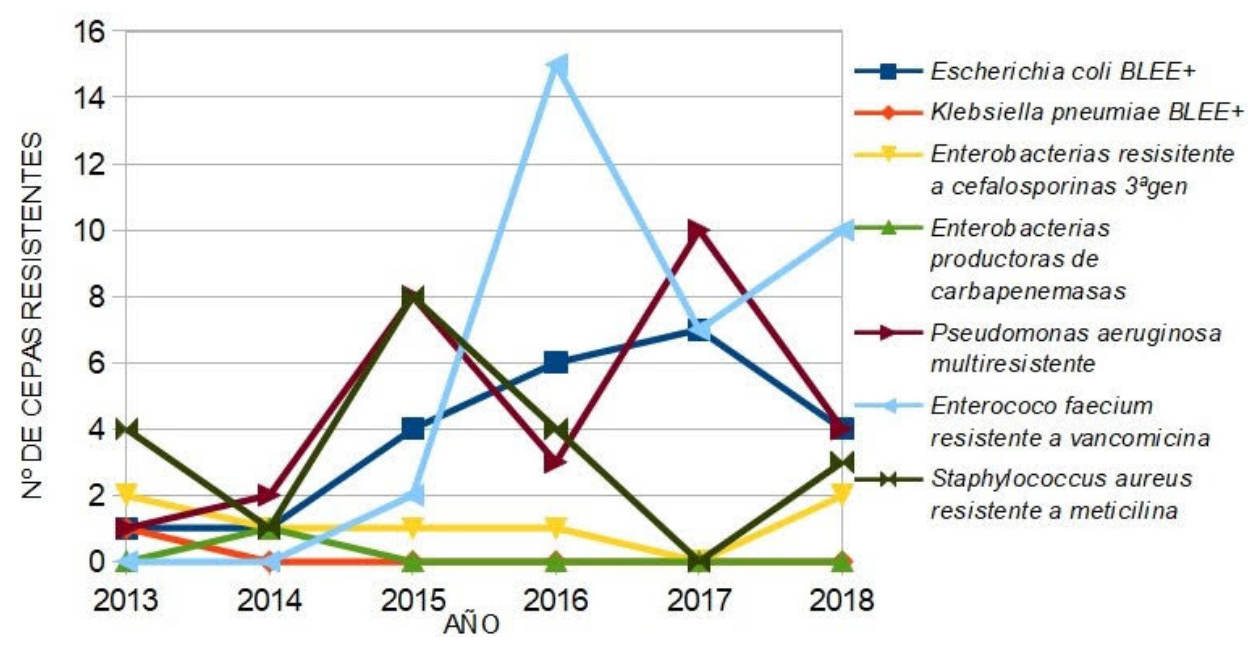

Figura 3: Progresión de bacterias resistentes por año. 


\section{Discusión}

De acuerdo con las conclusiones principales del primer informe Jiacra España (AEMPS, 2018), elaborado en el marco del Plan Nacional frente a la Resistencia de Antibióticos (PRAN) del Ministerio de Sanidad, en el ámbito hospitalario se ha registrado una subida del consumo de antibióticos del 9,5\% desde el 2012; este dato concuerda con la tendencia reflejada por los datos recogidos de la UCI de un aumento del 11,51 \% en el hospital de El Bierzo.

Según una nota informativa de la Agencia Española del Medicamento y Productos Sanitarios (AEMPS), la tendencia creciente del consumo de antibióticos desde 2012 se estabilizó en 2015, con un ralentizado incremento entre 2016 y 2017, y que podría tener relación con el aumento de resistencias a antibióticos. Estos datos nacionales encajan con las tendencias de consumo del hospital de El Bierzo. El abordaje de este problema, según Cristina Muñoz Madero, una de las coordinadoras del PRAN, debe ser desde un punto de vista multidisciplinar, como lo es la implantación de equipos PROA en los hospitales (Redacción Médica, 2018; AEMPS, 2018).

El uso de las DDD y DDD/100 estancias permitió hacer comparaciones entre los años estudiados, sin que los datos se vean afectados por los cambios de precios o presentaciones comerciales, sin embargo, la DDD no refleja necesariamente la dosis diaria recomendada o prescrita, pues estas son individualizadas, especialmente en pacientes críticos. Por lo que los datos de DDD son una estimación aproximada y no una fotografía real (Vega et al., 2015), aunque sí permiten comparar el consumo del servicio estudiado con otros servicios u otros hospitales del mismo nivel.

Cabe destacar que el consumo de ciertos principios activos se mantiene durante los seis años elevado, llegando a copar la práctica totalidad del consumo de antibióticos los últimos tres años. El consumo de linezolid es alto todos los años, oscilando entre 8,66 \% y 13,36\% del total, lo cual es llamativo, pues es un antibiótico de uso restringido. También hacer mención a la disminución del uso de cotrimazol, debido a las múltiples resistencias que se han registrado.

Respecto a las infecciones por $\operatorname{GRAM}(+)$, avala la tendencia al alza del consumo de linezolid, así como de cloxaciclina, cuyo consumo aumenta, al mismo tiempo que se mantiene el de ampicilina, lo que refleja un aumento del empleo de betalactamas en general.

Así como los antibacterianos relacionados con infecciones por GRAM(+), exceptuando linezolid, mantienen valores relativamente bajos, en los asociados a infecciones por GRAM(-) ocurre lo contrario, las tendencias de consumo en los más empleados, aminoglucósidos, carbapenems, cefalosporinas de $3 .^{a}$ y $4 .^{a}$, amoxicilina/clavulánico y piperacilina/tazobactam, son al alza, y en los otros se mantienen estables. 
Respecto a otros antibacterianos y antimicóticos, todas las tendencias son ligeramente al alza, excepto la de las fluoroquinolonas, que refleja un leve descenso en su consumo desde 2015. Se debe indicar que el valor de antimicóticos de 2015 es un valor aberrante debido a un elevado consumo de anfotericina B sin trascendencia para el estudio.

Los aislamientos de los seis años reflejan que el número de microorganismos aislados ha aumentado para todas las especies de 2013 a 2018, con un incremento del $86 \%$ de aislamientos del inicio al fin del estudio.

Del total de las cepas aisladas, un $67 \%$ corresponde a microorganismos GRAM(-), siendo la más presente $E$. coli, y el $33 \%$ restante a GRAM(+), dentro de las cuales Enterococo faecium y $S$. aureus son las más importantes.

Este patrón de aislamientos se corresponde con el patrón de consumo, en el que los antibióticos para GRAM(+) corresponden a un tercio de los destinados a infecciones por GRAM(-), siendo esta la proporción de aislamientos GRAM(+)/ GRAM(-) de 2013-2018.

Al fijarnos en el patrón de resistencias, también se puede apreciar que las cepas resistentes a GRAM(-) y GRAM(+) han aumentado en los últimos años, siendo de entre los GRAM(-) E. coli y $P$. aeruginosa las que han visto aumentado su número de cepas resistentes y en GRAM(+) tanto Enterococo resistente a vancomicina como $S$. aureous meticilin-resisitente (SAMR).

\section{Conclusión}

Con estos datos, podemos concluir que el consumo de antibióticos de la UCI sigue, en general, la tendencia nacional. Pero si comparamos los datos de los estudios ENVIN nacionales con los de ENVIN de la UCI del hospital, se observó que la tendencia de días sin ATB/estancias totales ha sido un poco mayor en nuestra UCI de estudio que en las UCI a nivel nacional (SEMICYUC, 2020).

Sin embargo, sería aconsejable realizar futuros estudios empleando otras unidades de consumo, como son las Dosis Diarias Prescritas (DDP) o Dosis Diaria de Tratamiento (DDT), para obtener una visión más objetiva y real de la situación del consumo en la UCI, así como datos sobre la adecuación de las prescripciones e indicaciones para las que se emplean los antibióticos (Álvarez-Lerma et al., 2018).

El consumo de cloxaciclina se relaciona con el tratamiento en monoterapia de bacteriemias por $S$. aureus meticilin-sensible y en biterapia con glucopéptidos o lipopéptidos para SAMR.

Según las últimas guías un consumo de linezolid por encima de 12 DDD se acompaña de la aparición de Staphylococcus coagulasa negativo resistentes a linezolid (Rodríguez-Lucas et al., 2018). Dado que la pauta inicial para el tratamiento empírico del shock séptico, en las UCI españolas, es de meropenem y linezolid 
y su elevado consumo se relaciona con la aparición de resistencias, hay que tener en cuenta los elevados valores de estos antibióticos para futuros estudios de resistencias, pudiendo demostrar que esta combinación, a largo plazo, constituye una mala praxis.

El aumento endémico de cepas resistentes de $E$. faecium se debe a un brote monoclonal nosocomial que se inició en 2016, por lo que son cepas endémicas del hospital.

Por esto último, se recomienda la necesidad de la adaptación de las guías de antibioticoterapia a las infecciones más frecuentes, incluyendo los antibacterianos más adecuados, para así optimizar el tiempo de duración de tratamiento y la profilaxis antibacteriana (Giachetto et al., 2003; Guzmán-Terán, 2018).

Como conclusión, es necesario hacer estudios de adecuación de antimicrobianos empleando DDP y recalcar la necesidad de establecer los parámetros en los que se desarrollará el equipo PROA-UCI, estableciendo unos objetivos de consumo y flora de los que se haga un seguimiento regular, partiendo de los datos obtenidos en el presente análisis.

\section{BibliografíA}

AEMPS. Informe JIACRA-España [Internet]. 06/18. [citado 22 de febrero de 2020]. p. 165. Disponible en: http://resistenciaantibioticos.es/es/publicaciones

AEMPS. El consumo total de antibióticos en salud humana bajó un 4,34 \% entre 2016 y 2017. 2018 jun. p. 2. Report No.: AEMPS, 10/2018.

Álvarez-Lerma F, Grau S, Echeverría-Esnal D, Martínez-Alonso M, Gracia-Arnillas MP, Horcajada JP et al. A Before-and-After Study of the Effectiveness of an Antimicrobial Stewardship Program in Critical Care. Antimicrob Agents Chemother [Internet]. 16 de enero de 2018. [citado 14 de febrero de 2020]; 62(4). Disponible en: http://aac. asm.org/lookup/doi/10.1128/AAC.01825-17

Contreras JR. Consumo de antibióticos y prevención de las resistencias bacterianas. Rev Pediatr Aten Primaria Supl. 2018; (27):13-21.

Generalitat de Catalunya. La implantación de un PROA en una UCI de Cataluña reduce el consumo de antibióticos [Internet]. Seguridad de los Pacientes. [citado 5 de noviembre de 2019]. Disponible en: http://seguretatdelspacients.gencat.cat/es/ detalls/noticia/La-implementacio-dun-PROA-a-una-UCI-de-Catalunya-redueixel-consum-dantibiotics

Giachetto DG, Martínez A, Pírez MC, Algorta G, Banchero P, Camacho G et al. Vigilancia del uso de antibióticos en el Hospital Pediátrico del Centro Hospitalario Pereira Rossell: susceptibilidad antimicrobiana; gasto y consumo de antibióticos. 2003;19:8.

Guzmán-Terán C. Análisis de usos y resistencia a antibióticos en una UCI de Montería, Colombia. Rev Méd Risaralda. 2018; 24(2):75-80. 
Lázaro E, Oteo J. Evolución del consumo y de la resistencia a antibióticos en España. Inf Ter Sist Nac Salud. 2006; 30:10-19.

Pallares CJ, Martínez E. Implementación de un programa de uso regulado de antibióticos en 2 unidades de cuidado intensivo médico-quirúrgico en un hospital universitario de tercer nivel en Colombia. Infectio. 2012; 16(4):192-198.

Redacción. El consumo de antibióticos en hospitales ha crecido un 9,5\% desde 2012 [Internet]. Redacción Médica. [citado 5 de noviembre de 2019]. Disponible en: https://www.redaccionmedica.com/secciones/farmacia/ el-consumo-de-antibioticosen-hospitales-ha-crecido-un-9-5-desde-2012-2879

Rodríguez-Baño J, Paño-Pardo JR, Álvarez-Rocha L, Asensio Á, Calbo E, Cercenado E et al. Programas de optimización de uso de antimicrobianos (PROA) en hospitales españoles: documento de consenso GEIH-SEIMC, SEFH y SEMPSPH. Farm Hosp. enero de 2012; 36(1): 33.e1-33.e30.

Rodríguez-Lucas C, Rodicio MR, Càmara J, Domínguez MÁ, Alaguero M, Fernández J. Long-term endemic situation caused by a linezolid- and meticillinresistant clone of Staphylococcus epidermidis in a tertiary hospital. J Hosp Infect [Internet]. octubre de 2019. [citado 20 de febrero de 2020]. Disponible en: https://linkinghub.elsevier. com/retrieve/pii/S0195670119304487

SEMICYUC [Internet]. ENVIN-HELICS. [citado 22 de febrero de 2020]. Disponible en: http://hws.vhebron.net/envin-helics/

Vega EM, Fontana D, Iturrieta M, Segovia L, Rodríguez G, Agüero S. Consumo de antimicrobianos en la Unidad de Terapia Intensiva del Hospital Dr. Guillermo Rawson-San Juan, Argentina. Rev Chil Infectol. junio de 2015; 32(3):259-265.

WHOCC - ATC/DDD Index [Internet]. WHO Collaborating Centr for Drugs Statistics Methodology. [actualizado marzo de 2019; citado 15 de febrero de 2020]. Disponible en: https://www.whocc.no/atc_ddd_index/?code=J01E\&showdescription=no 